\title{
The Relationship between Crystal Rotation Axis Orientation and Active Slip System in Pure Aluminum Tricrystal Deformed in Compression
}

\author{
Keizo Kashihara \\ Wakayama National College of Technology, Gobo 644-0023, Japan
}

The relationship between crystal rotation axis orientations and active slip systems was investigated in an aluminum tricrystal deformed under compression to a strain of 0.15 . Schmid factors of slip systems were examined for three component crystals. After compression, slip bands near a triple junction were observed using a scanning electron microscope. Crystal rotation axis orientations were calculated by comparison of the crystal orientations before and after deformation. One of the three component crystals yielded various crystal rotation axis orientations. The active slip systems in the crystal, which generate crystal rotation relative to the initial orientation, were estimated from the slip bands, crystal rotation axis orientations, Schmid factors and $\langle 112\rangle$ lattice rotation axis orientations. Here, the lattice rotation axis orientations are assigned to individual slip systems, since they operate toward $\langle 110\rangle$ directions on $\{111\}$ planes. Based on the estimation of active slip systems, in an area far from the triple junction of the crystal, the crystal rotation relative to the initial orientation was introduced by imbalance operation of the primary and additional slip systems. In the vicinity of the triple junction, the crystal rotation was generated by three slip systems with different amounts of slip operations. The two additional slip systems on the same slip plane were partially activated along a straight boundary.

[doi:10.2320/matertrans.MBW200704]

(Received October 2, 2007; Accepted October 24, 2007; Published December 5, 2007)

Keywords: aluminum, tricrystal, deformation, slip system, lattice rotation, crystal rotation

\section{Introduction}

The development of deformation structure is closely related to the activated slip systems. Inhomogeneous deformation is introduced by the deference of active slip systems, which occurs generally in the interior of grains, at grain boundaries and in deformation zones around second phase particles. Deformation inhomogeneity is recognized by slip band observations and crystal orientation measurements on a specimen surface.

Using electron back-scatter diffraction pattern (EBSP) measurements, Wert and co-workers established a method to predict the slip systems activated in deformation by comparing the crystal rotation axis orientation and the shear amplitude of slip systems. ${ }^{1-5)}$ The crystal rotation axis (CRA) orientation at an EBSP data point is obtained by the comparison of crystal orientations before and after deformation. Since the CRA orientation changes depending on not only the kind of active slip system but also the ratio of their operations, the development of deformation structure by the slip operations can be understood in detail. This prediction method has been applied to cold-rolled aluminum single crystals with various initial orientations, ${ }^{1)}$ aluminum single crystal deformed in tension ${ }^{2,4)}$ and polycrystalline copper deformed in tension. ${ }^{5)}$

Simplifying this method, Kashihara and Wert examined slip systems activated near a grain boundary in an aluminum bicrystal. ${ }^{6)}$ Active slip systems were estimated by comparing the CRA orientation and the $\langle 112\rangle$ lattice rotation axis (LRA) orientations. The LRA orientations are geometrically assigned to individual slip systems in aluminum, since they operate toward the $\langle 110\rangle$ directions on $\{111\}$ planes under an applied stress.

In the present study, in order to verify the validity of the prediction of active slip systems, slip systems activated near a triple junction of an aluminum tricrystal deformed in compression were examined using the prediction method proposed in the previous study.

\section{Experimental Method}

A cylindrical aluminum tricrystal (99.99\% purity) was grown in the size of $\phi 20 \times 50 \mathrm{~mm}^{3}$ by a modified Bridgman method. $4 \mathrm{~mm}$ thick disks were cut from the tricrystal, as shown in Fig. 1(a). A specimen for the compression test was cut using an electric discharge machine, and then mechanically and electrolytically polished. The test part of the specimen was $7 \mathrm{~mm}$ in length, $6 \mathrm{~mm}$ in width and $3.6 \mathrm{~mm}$ in thickness (Fig. 1(b)). The three component crystals are termed Crystal A, B and C.

Figure 2 shows the initial orientations of Crystals A, B and $\mathrm{C}$ analyzed by the electron channeling pattern (ECP) method. The names of the twelve slip systems follow the Bishop-Hill notation. ${ }^{7)}$ Slip plane normals, slip directions and Schmid factors of the slip systems are shown in Table 1.

The specimen was deformed in compression to a true strain of 0.15 at a strain rate of $4 \times 10^{-4} \mathrm{~s}^{-1}$. After compression testing at room temperature, slip band observations were carried out using a scanning electron microscope in order to (a)

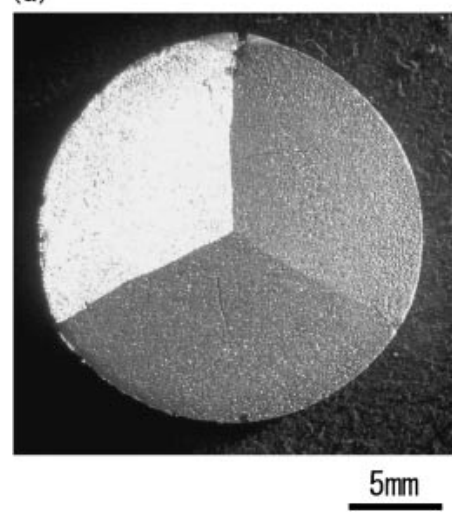

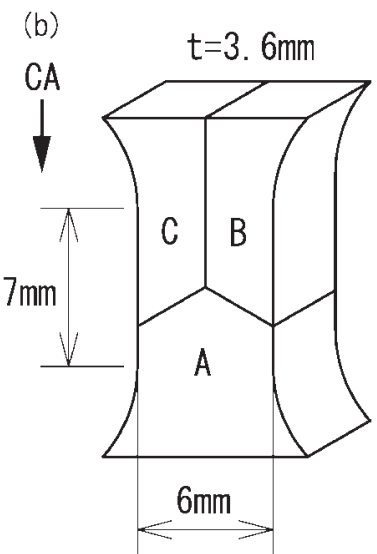

Fig. 1 (a) A disk cut from a cylindrical aluminum tricrystal and (b) the specimen fabricated and used for the compression test. 

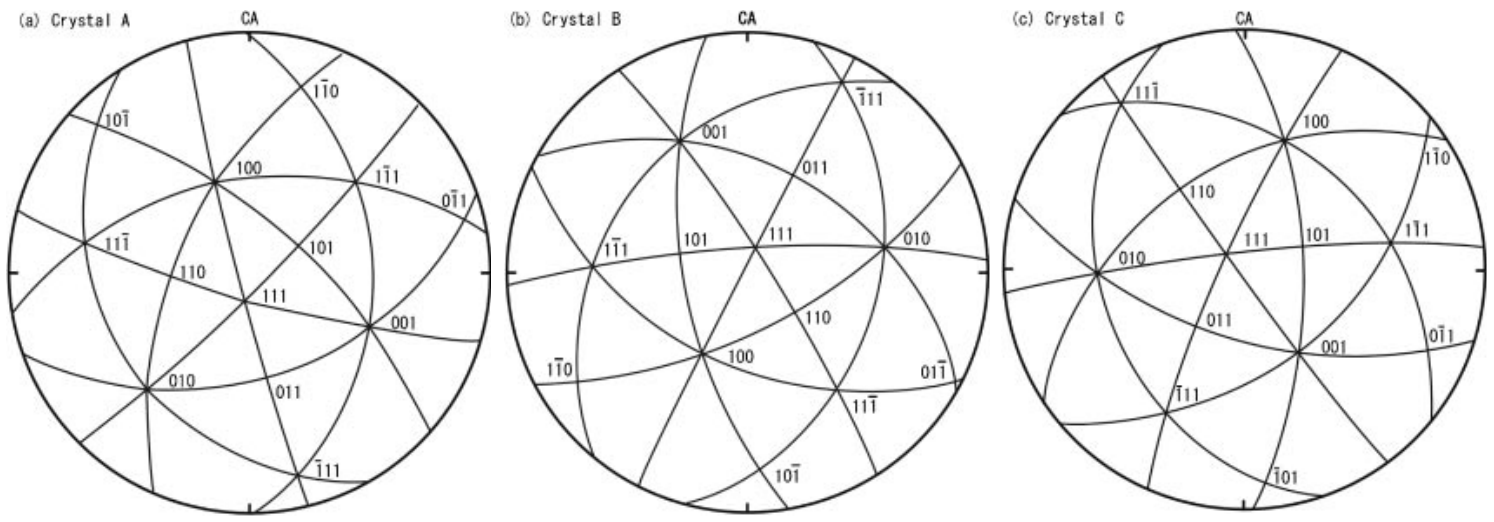

Fig. 2 Initial orientations of (a) Crystal A, (b) Crystal B and (c) Crystal C. CA indicates the compression axis.

Table 1 Slip systems of the component crystals and their Schmid factors.

\begin{tabular}{cccccc}
\hline \multirow{2}{*}{$\begin{array}{c}\text { Slip } \\
\text { system }\end{array}$} & Slip plane & \multirow{2}{*}{$\begin{array}{c}\text { Slip } \\
\text { direction }\end{array}$} & \multicolumn{3}{c}{ Schmid factor } \\
\cline { 5 - 6 } a1 & 111 & $01 \overline{1}$ & Crystal A & Crystal B & Crystal C \\
a2 & 111 & $\overline{1} 0.05$ & 0.10 & 0.06 \\
a3 & 111 & $1 \overline{1} 0$ & 0.19 & 0.10 & 0.08 \\
b1 & $\overline{1} \overline{1} 1$ & $0 \overline{1} \overline{1}$ & 0.14 & 0.48 & 0.34 \\
b2 & $\overline{1} \overline{1} 1$ & 101 & 0.04 & 0.13 & 0.11 \\
b3 & $\overline{1} \overline{1} 1$ & $\overline{1} 10$ & 0.18 & 0.35 & 0.45 \\
c1 & $\overline{1} 11$ & $01 \overline{1}$ & 0.22 & 0.42 & 0.34 \\
c2 & $\overline{1} 11$ & 101 & 0.20 & 0.15 & 0.11 \\
c3 & $\overline{1} 11$ & $\overline{1} \overline{1} 0$ & 0.00 & 0.27 & 0.46 \\
d1 & $1 \overline{1} 1$ & $0 \overline{1} \overline{1}$ & 0.40 & 0.05 & 0.05 \\
d2 & $1 \overline{1} 1$ & $\overline{1} 01$ & 0.40 & 0.07 & 0.12 \\
d3 & $1 \overline{1} 1$ & 110 & 0.00 & 0.02 & 0.07 \\
\hline
\end{tabular}

determine the slip planes on which the slip systems operate. Furthermore, crystal orientations near the triple junction after deformation were measured by the electron back-scatter diffraction pattern (EBSP) method. This method yields the Euler angle for crystal orientation. The EBSP was obtained at $1 \mu \mathrm{m}$ intervals on a square area of $200 \times 160 \mu \mathrm{m}^{2}$. The EBSP data points on the area were about 32000 .

The CRA orientation was obtained from the rotation matrix $G,{ }^{8}$ )

$$
G=S A_{2} A_{1}^{-1}=\left[\begin{array}{lll}
g_{11} & g_{12} & g_{13} \\
g_{21} & g_{22} & g_{23} \\
g_{31} & g_{32} & g_{33}
\end{array}\right]
$$

where $S$ is the symmetry matrix having 24 different patterns in cubic materials. $A_{1}$ and $A_{2}$ are the orientation matrices for crystal orientations before and after deformation, respectively.

The rotation angle $\theta$ between the orientations before and after deformation is given by

$$
\theta=\cos ^{-1}\left[\frac{\boldsymbol{g}_{11}+\boldsymbol{g}_{22}+\boldsymbol{g}_{33}-1}{2}\right]
$$

The minimum value among 24 rotation angles is adopted as the correct rotation angle. Then, the CRA orientation of $\left[d_{1}, d_{2}, d_{3}\right]$ in the rotation angle is represented by

$$
\left[d_{1}, d_{2}, d_{3}\right]=\left[\frac{\boldsymbol{g}_{32}-\boldsymbol{g}_{23}}{2 \sin \theta}, \frac{\boldsymbol{g}_{13}-\boldsymbol{g}_{31}}{2 \sin \theta}, \frac{\boldsymbol{g}_{21}-\boldsymbol{g}_{12}}{2 \sin \theta}\right]
$$

Here, $d_{1}^{2}+d_{2}^{2}+d_{3}^{2}$ is equal to 1 .

A CRA orientation map was constructed using the method proposed by Wert. ${ }^{1)}$ This map shows the CRA orientations at the EBSP data points, which are represented following the color-coding shown in Fig. 5(b). If the crystal orientation after deformation at a data point rotates around [100] or [100] relative to the initial orientation, this point is colored red on the map. Data points colored blue and green represent crystal rotations around [010] or [010] and [001] or [001] , respectively. In this study, a data point is colored white when the rotation angle relative to the initial orientation is less than $3^{\circ}$.

\section{Experimental Results}

\subsection{Slip band observations}

Figure 3 shows an SEM micrograph taken at a low magnification. The interiors of Crystal A, B and C reveal slip bands parallel to (1)11), ( $\overline{1} \overline{1} 1)$ and ( $\overline{1} 11)$, respectively. These are the main slip bands. The main slip bands correspond to the slip planes to which the slip system having the highest Schmid factor belonged. Additional slip bands were observed near the triple junction.

A magnified SEM micrograph is shown in Fig. 4. In Crystal A, additional slip bands parallel to (111) are observed across the main slip bands parallel to (111). In Crystal C, additional slips of (111) were found across the main slip bands of (111). The additional slip bands seen in Crystal C appear to be connected to the main slip bands in Crystal B. Therefore, we consider that slip transfers between them occur across the grain boundaries. This will be discussed later in section 4.3 .

\subsection{EBSP analysis}

Figure 5(a) shows the CRA orientation map. This map is represented at the same magnification as the slip band structure shown in Fig. 4. As mentioned above, the CRA orientations on the map were colored for each EBSP data point. The CRA orientations can be identified based on the color-coding shown in Fig. 5(b). It is obvious that Crystal C has a variety in color compared to Crystals A and B. In Crystal $\mathrm{C}$, region 1 away from the triple junction is colored 


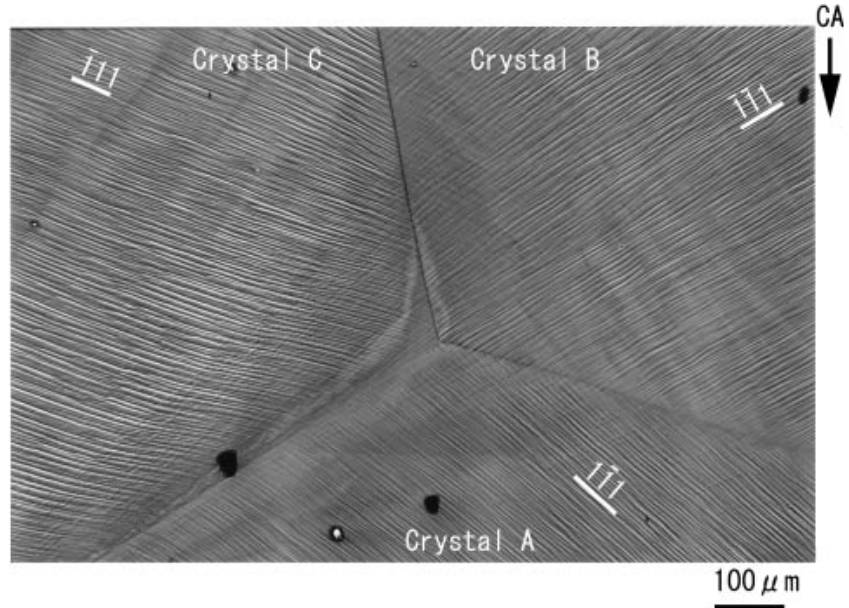

Fig. 3 SEM micrograph of the specimen after compression taken at a low magnification.

red, whereas region 2 in the vicinity of the triple junction is colored blue. Since the CRA orientation in region 2 was different from that in region 1 , it was evident that region 2 was the affected zone in deformation by the triple junction. The transition region between regions 1 and 2 was observed to have alternate red and blue patterns. The green regions, such as region 3 , were only formed along the grain boundary between Crystals B and C. Thus, this map indicates that different slip in deformation occurs depending on the location in Crystal C.

\section{Discussion}

We now discuss the slip systems activated in Crystal C, which showed various CRA orientations. Basically, deformation of a single crystal sample involves simple shear and rigid-body rotation. If rigid-body rotation is taken out of the deformed sample, the remainder is the lattice rotation, which results from the simple shear introduced by the operation of slip systems. In FCC metals with high stacking fault energy, such as aluminum, since the slip systems operate toward $\langle 110\rangle$ directions on $\{111\}$ planes, the lattice rotation axis (LRA) orientations by the slip become $\langle 112\rangle$. Slip systems have individual LRA orientations. Their rotations (clockwise or counter-clockwise) are determined by the geometric relationship between the external force and the slip systems. The LRA orientations of twelve slip systems in Crystal C are listed in Table 2. In this study, the LRA orientations were regarded as unit vectors. These vectors were combined so as to match the CRA orientation actually observed in the CRA orientation map. From the combination of vectors, we can estimate the active slip systems which generate the crystal rotation relative to the initial orientation. As shown in Fig. 4, in Crystal C, the main slip bands parallel to (111) as well as additional slip bands parallel to (111) were observed. There are three slip systems, $\mathrm{c} 1, \mathrm{c} 2$ and $\mathrm{c} 3$, on (111), whereas the b1, b2 and b3 systems are on (111). Since the slip systems having a higher Schmid factor tend to be more activated, the four slip systems, c1, c3, b1 and b3, are selected primarily for the discussion. The geometrical CRA orientations calculated

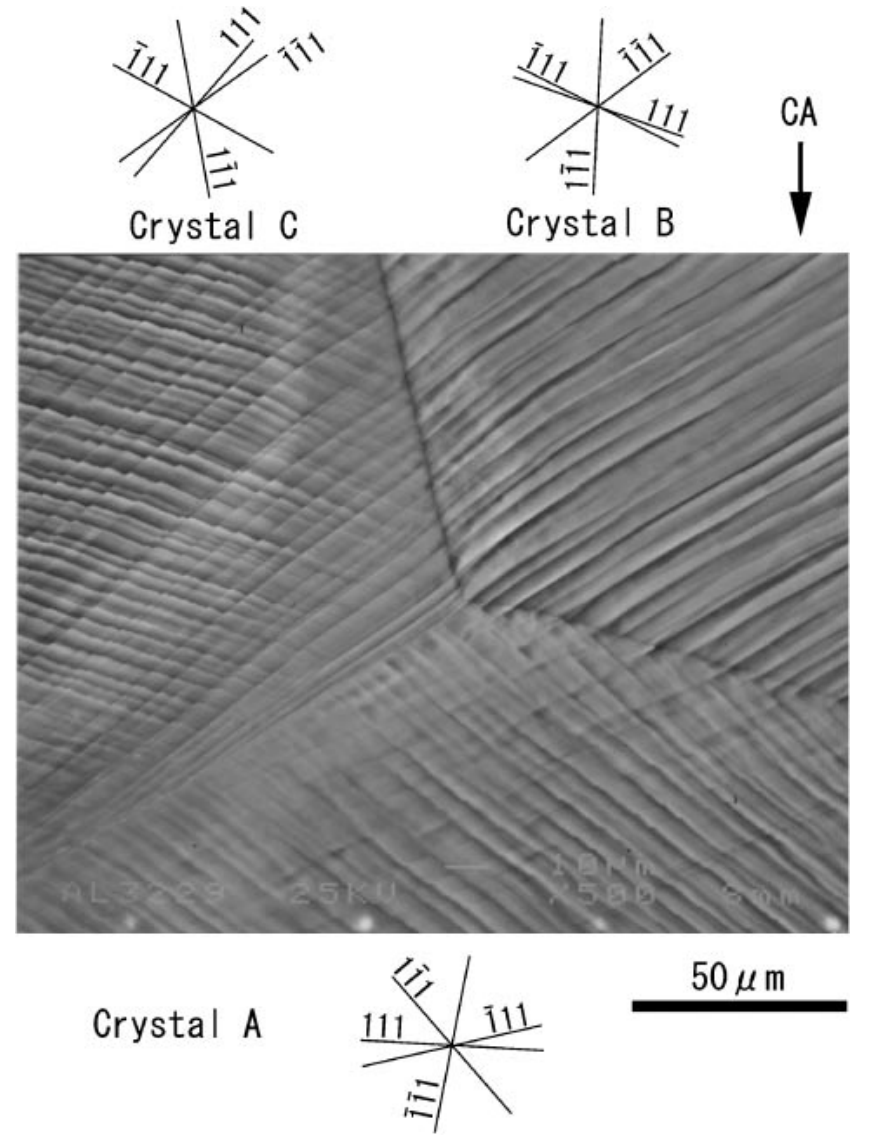

Fig. 4 SEM micrograph of the specimen after compression obtained using high magnification.

from the LRA orientations are compared to the CRA orientations actually observed.

\subsection{Region 1 far from the triple junction}

Figure 6(a) shows the CRA orientations analyzed at 14 data points of region 1 in Fig. 5(a). In Fig. 4, double slips of (111) and ( $\overline{1} \overline{1} 1)$ occurred. The CRA orientations indicated by red dots are located at orientations around [310]. Table 3 yields the geometrical CRA orientation calculated from the LRA orientations when the corresponding slip systems operate equivalently. The geometrical CRA orientations obtained from the combination of the LRA orientations did not coincide with the CRA orientation actually observed. So, the ratio of the operation of slip systems was changed. This means that an imbalance operation of slip systems occurs. When the c3 and b1 slip systems are activated, and in addition, the ratio of the operation of $c 3$ to that of $b 1$ is $1: 2$, then the geometrical CRA orientation becomes $[\overline{3} 10]$. Therefore, the crystal rotation of region 1 relative to the initial orientation was introduced by the imbalance slip operation of c3 on (111) and b1 on ( $\overline{1} \overline{1} 1)$.

\subsection{Region 2 near the triple junction}

Region 2 near the triple junction of Crystal $\mathrm{C}$ revealed double slip of (111) and ( $\overline{1} \overline{1} 1)$ as shown in Fig. 4, whereas region 2 had a different color compared to region 1 as shown in Fig. 5(a). It is obvious that region 2 is the affected zone in deformation due to the triple junction. The CRA orientations 
(a)

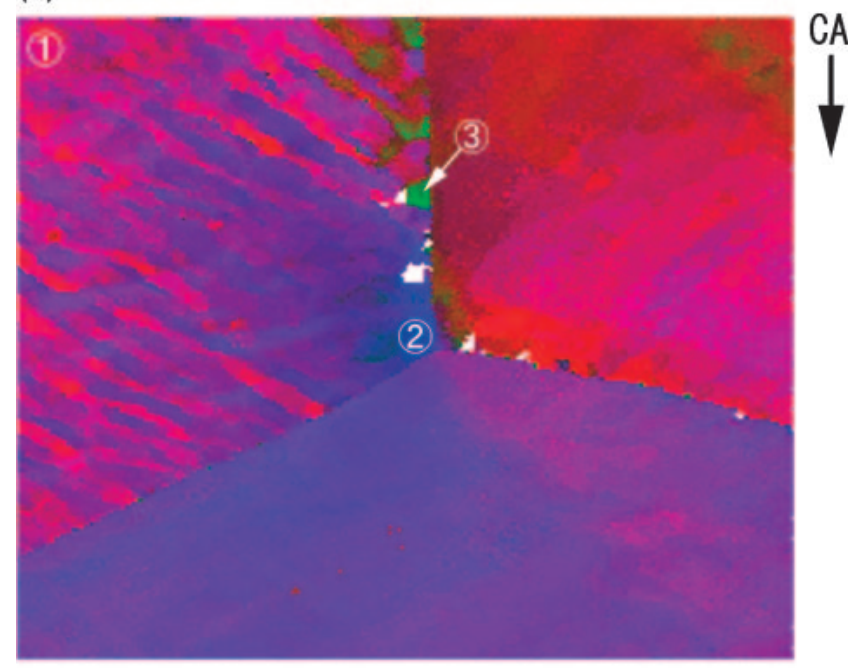

(b)

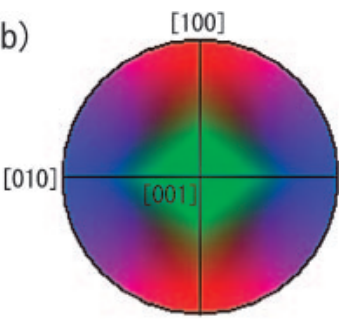

Fig. 5 (a) Crystal rotation axis (CRA) orientation map and (b) colorcoding projection for crystal orientations.

analyzed at 27 data points are shown in Fig. 6(b). The CRA orientations indicated by the red dots are located at orientations around [021]. As shown in Table 3, the CRA orientation of [021] did not coincide with the geometrical CRA orientations calculated from the equivalent operations of slip systems. However, the geometrical CRA orientation becomes [053] when the b1, c1 and c3 slip systems operate in the ratio of $4: 3: 2$. Therefore, the deformation near the triple junction likely progresses by different amounts of slip operations of b1, c1 and c3, and these operations generate crystal rotation relative to the initial orientation.

The kinds of slip systems operated in region 2 differed from those in region 1 . In region 2 , c1 slip system was additionally activated due to the effect of triple junction. Here, $\mathrm{c} 1$ is the coplanar slip system to $\mathrm{c} 3$ primary slip system. The alternate red and blue patterns observed in the transition
Table 2 Lattice rotation axis (LRA) orientations and Schmid factors of the slip systems in Crystal C.

\begin{tabular}{cccccc}
\hline $\begin{array}{c}\text { Slip } \\
\text { system }\end{array}$ & $\begin{array}{c}\text { Slip } \\
\text { plane }\end{array}$ & $\begin{array}{c}\text { Slip } \\
\text { direction }\end{array}$ & $\begin{array}{c}\text { LRA } \\
\text { (clockwise) }\end{array}$ & $\begin{array}{c}\text { LRA } \\
\text { (counter } \\
\text { clockwise) }\end{array}$ & $\begin{array}{c}\text { Schmid } \\
\text { factor }\end{array}$ \\
\hline a1 & 111 & $01 \overline{1}$ & & $\overline{2} 11$ & 0.06 \\
a2 & 111 & $\overline{1} 01$ & $\overline{1} 2 \overline{1}$ & & 0.14 \\
a3 & 111 & $1 \overline{1} 0$ & & $11 \overline{2}$ & 0.08 \\
b1 & $\overline{1} \overline{1} 1$ & $0 \overline{1} \overline{1}$ & $\overline{2} 1 \overline{1}$ & & 0.34 \\
b2 & $\overline{1} \overline{1} 1$ & 101 & $1 \overline{2} \overline{1}$ & & 0.11 \\
b3 & $\overline{1} \overline{1} 1$ & $\overline{1} 10$ & & $\overline{1} \overline{1} \overline{2}$ & 0.45 \\
c1 & $\overline{1} 11$ & $01 \overline{1}$ & 211 & & 0.34 \\
c2 & $\overline{1} 11$ & 101 & $\overline{1} \overline{1} 1$ & & 0.11 \\
c3 & $\overline{1} 11$ & $\overline{1} \overline{1} \overline{1} 1$ & & $1 \overline{1} 2$ & 0.46 \\
d1 & $1 \overline{1} 1$ & $0 \overline{1} \overline{1}$ & & $21 \overline{1}$ & 0.05 \\
d2 & $1 \overline{1} 1$ & $\overline{1} 01$ & 121 & & 0.12 \\
d3 & $1 \overline{1} 1$ & 110 & & $\overline{1} 12$ & 0.07 \\
\hline
\end{tabular}

Table 3 Combination of slip systems and geometrical crystal rotation axis (CRA) orientations.

\begin{tabular}{lclc}
\hline $\begin{array}{c}\text { Combination of } \\
\text { slip systems }\end{array}$ & $\begin{array}{c}\text { Geometrical } \\
\text { CRA }\end{array}$ & $\begin{array}{c}\text { Combination of } \\
\text { slip systems }\end{array}$ & $\begin{array}{c}\text { Geometrical } \\
\text { CRA }\end{array}$ \\
\hline $\mathrm{b} 1+\mathrm{b} 3$ & $\overline{1} 0 \overline{1}$ & $\mathrm{c} 1+\mathrm{c} 3$ & 101 \\
$\mathrm{~b} 1+\mathrm{c} 1$ & 010 & $\mathrm{~b} 1+\mathrm{b} 3+\mathrm{c} 1$ & $\overline{1} 1 \overline{2}$ \\
$\mathrm{~b} 1+\mathrm{c} 3$ & $\overline{1} 01$ & $\mathrm{~b} 1+\mathrm{c} 1+\mathrm{c} 3$ & 112 \\
$\mathrm{~b} 3+\mathrm{c} 1$ & $10 \overline{1}$ & $\mathrm{~b} 3+\mathrm{c} 1+\mathrm{c} 3$ & $2 \overline{1} 1$ \\
$\mathrm{~b} 3+\mathrm{c} 3$ & $0 \overline{1} 0$ & $\mathrm{~b} 1+\mathrm{b} 3+\mathrm{c} 3$ & $\overline{2} \overline{1} \overline{1}$ \\
\hline
\end{tabular}

region between regions 1 and 2 were attributed to the additional operation of $\mathrm{c} 1$.

The appearance of alternate patterns was similar to that of primary and coplanar slip zones, which was observed by etch pit method in tensile-deformed copper alloy single crystals. ${ }^{9)}$ The coplanar slip zones were formed by the banded accumulation of coplanar slip dislocations, which occurred so as to reduce the work hardening enhanced by primary slip dislocations. The etch pit patterns formed by the primary and coplanar dislocations appeared to band structure. In this study, we speculate that c1 slip system was originally activated near the triple junction, and the operation of $\mathrm{c} 1$ made the alternate red and blue patterns in the transition region, which were due to the interaction between $\mathrm{c} 1$ and $\mathrm{c} 3$ slip systems.
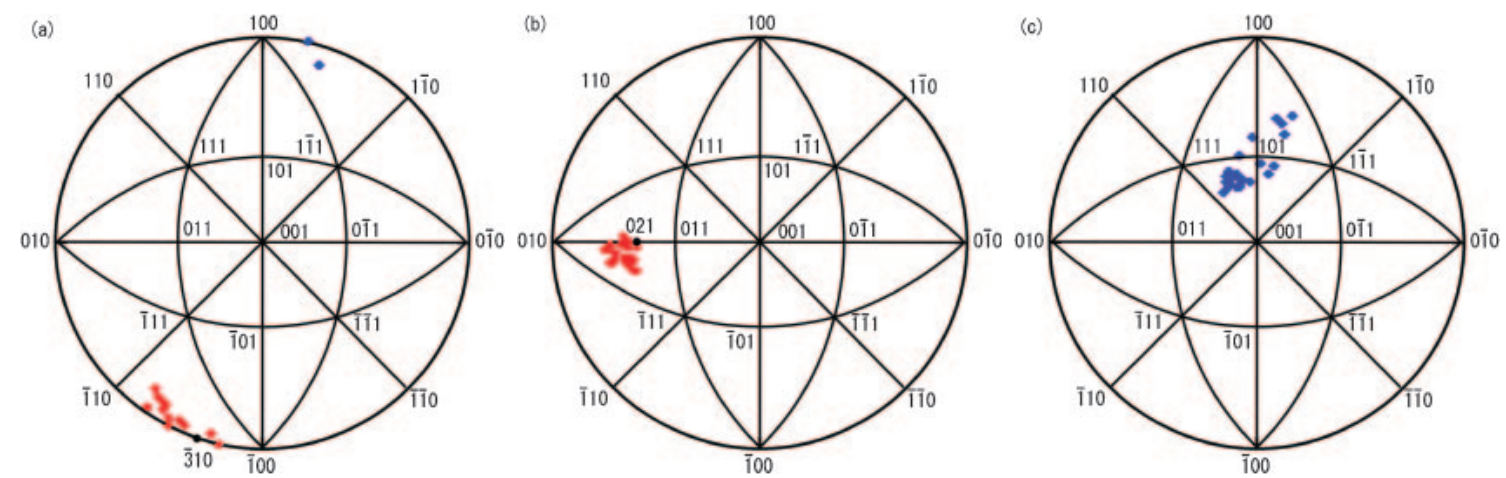

Fig. 6 CRA orientations of (a) Crystal A, (b) Crystal B and (c) Crystal C. Red and blue dots indicate clockwise and counter-clockwise rotations, respectively. 
Table 4 Transmission factor $\left(N_{\mathrm{ij}}\right)$ for the combination of the primary slip system of b1 in Crystal B and the slip systems of b1, b3, c1 and c3 with high Schmid factors in Crystal C.

\begin{tabular}{ccc}
\hline Crystal B & Crystal C & $N_{\mathrm{ij}}$ \\
\hline b1 & b1 & 0.13 \\
b1 & b3 & 0.52 \\
b1 & c1 & 0.12 \\
b1 & c3 & 0.49 \\
\hline
\end{tabular}

\subsection{Region 3 near the straight grain boundary}

Based on the slip bands observed in Fig. 3 and the Schmid factor listed in Table 1, it is evident that b1 in Crystal B and c3 in Crystal $\mathrm{C}$ are the primary slip systems. The CRA orientation map (Fig. 5) clearly reveals the occurrence of inhomogeneous deformation along the grain boundary between Crystals B and C. The typical example is region 3 in Crystal C. Since region 3 having green color was only observed along the grain boundary, region 3 is formed due to the strain incompatibility between Crystals B and C. The strain incompatibility should be canceled by the additional slips. The CRA orientations of region 3 are shown in Fig. 6(c). The CRA orientations (blue dots) at 27 data points are located at orientations around [ $\overline{1} 0 \overline{1}]$. Table 3 shows that the equivalent operation of $b 1$ and b3 results in the geometrical CRA orientation of [ $\overline{1} 0 \overline{1}]$. Therefore, it can be predicted that b1 and b3 operate equivalently in region 3 .

Slip operation in the vicinity of a straight grain boundary is often discussed using the stress transmission factor. ${ }^{10-14)}$ Here, the slip systems estimated from the analysis of the CRA orientation are compared with those predicted by the stress transmission factor, $N_{\mathrm{ij}}$. The factor $N_{\mathrm{ij}}$ indicates the degree of shear stress transfer from one crystal " $i$ " to another crystal " $\mathrm{j}$ ". It is quantitatively written as:

$$
N_{i j}=\left(\boldsymbol{e}_{i} \times \boldsymbol{e}_{j}\right)\left(\boldsymbol{g}_{i} \times \boldsymbol{g}_{j}\right)+\left(\boldsymbol{e}_{i} \times \boldsymbol{g}_{j}\right)\left(\boldsymbol{g}_{i} \times \boldsymbol{e}_{j}\right)
$$

where $e_{i}$ and $g_{i}$ are the slip plane normal and the slip direction of an incoming slip system (in the crystal $i$ ), and $e_{j}$ and $g_{j}$ are the slip plane normal and slip direction of the outgoing slip system (in the crystal $\mathrm{j}$ ). The outgoing slip system having the maximum $N_{\mathrm{ij}}$ is activated across the grain boundary. Based on the Schmid factor (Table 1) and the slip band formation (Fig. 4), $N_{\mathrm{ij}}$ was calculated in the combination of b1, b3, c1 and $\mathrm{c} 3$ in Crystal $\mathrm{C}$ as outgoing slip systems and b1 in Crystal $\mathrm{B}$ as an incoming slip system, because the slip bands to which these slip systems belonged were actually observed and in addition they had the higher Schmid factor. The value of $N_{\mathrm{ij}}$ is shown in Table 4. The pair of the b1 system in Crystal B and b3 in Crystal C had the highest $N_{\mathrm{ij}}$. The b3 slip system in Crystal $\mathrm{C}$ agrees with that estimated by the analysis of the CRA orientation. The second highest $N_{\mathrm{ij}}$ was for the pair of the b1 system in Crystal B and the c3 systems in Crystal C. These are the primary slip systems in the component crystals. We discuss the reason for the operation of b1 in Crystal C, which was obtained by the analysis of the CRA orientation. Macroscopically, strain incompatibility occurs between the primary slip system of $\mathrm{b} 1$ in Crystal $\mathrm{B}$ and that of $\mathrm{c} 3$ in
Crystal C. The incompatibility is canceled by additional slips systems. The operations of additional slip systems were not uniform, as shown in Fig. 5, and the specific region where the additional slip systems operated was the region 3. Based on both the $N_{\mathrm{ij}}$ and the CRA orientation analysis, it is obvious that the additional slip system primarily activated was b3 in Crystal C. The operation of b3 can diminish the strain incompatibility between Crystals B and C, but there is still the incompatibility left in Crystal $\mathrm{C}$. This has to be canceled by further operation of the other slip system. We speculate that the remaining incompatibility is canceled by b1 in Crystal C, which is the coplanar slip system to b3.

\section{Summary}

Crystal rotation axis (CRA) orientations near a triple junction in an aluminum tricrystal deformed in compression were investigated. A variety in the CRA orientation was especially observed in one of the component crystals (Crystal C). In a region far from the triple junction of Crystal C, crystal rotation relative to the initial orientation was introduced by the imbalance operations of the primary and additional slip systems. In the vicinity of the triple junction, the primary slip system and the two additional slip systems operate with different amounts of slip. The crystal rotation in the local region along the grain boundary is caused by the two additional slip systems. Thus, the slip systems, which generate crystal rotations relative to the initial orientation, can be estimated by the analysis of the CRA orientations combined with Schmid factors, slip bands and the lattice rotation axis orientations.

\section{Acknowledgements}

The author would like to thank T. Shibayanagi, T. Okada and K. Hosokawa for the use of SEM facility and the technical assistance. The work was performed with financial support from the Japan Aluminum Association.

\section{REFERENCES}

1) J. A. Wert: Acta Mater. 50 (2002) 3125-3139.

2) J. A. Wert, X. Huang and F. Inoko: Proc. R. Soc. Lond. A 459 (2003) 85-108.

3) Z. J. Li, A. Godfrey and Q. Liu: Acta Mater. 52 (2004) 149-160.

4) J. A. Wert, K. Kashihara, T. Okada, X. Huang and F. Inoko: Philos. Mag. 85 (2005) 1989-2006.

5) C. Thorninh, M. A. J. Somers and J. A. Wert: Mater. Sci. Eng. A397 (2005) 215-228.

6) K. Kashihara and J. A. Wert: Mater. Trans. 47 (2006) 233-238.

7) J. F. Bishop and R. Hill: Philo. Mag. 42 (1951) 1298-1307.

8) F. J. Humphreys and M. Hatherly: Recrystallization and Related Annealing Phenomena, (Pergamon Press 1996), p. 59.

9) J. Takamura: Bull. Japan Inst. Metals 25 (1986) 379-391.

10) J. D. Livingston and B. Chalmers: Acta Metall. 5 (1957) 322.

11) R. E. Hook and J. P. Hirth: Acta Metall. 15 (1967) 535.

12) F. Inoko, K. Akizono and G. Mima: J. Japan Inst. Metals 36 (1972) 380.

13) S. Miura and Y. Saeki: Acta Metall. 26 (1978) 93.

14) K. Kashihara and F. Inoko: Acta Metall. 49 (2001) 3051-3061. 